\title{
Successful Treatment of Intra-Arterial Peplomycin Infusion for Recurrent Oral Florid Papillomatosis
}

\author{
Atsushi Tanemura, Madoka Takafuji, Yuma Hanaoka, Eiji Kiyohara, Mari Wataya-Kaneda, \\ Manabu Fujimoto \\ Department of Dermatology, Osaka University Graduate School of Medicine, Suita, Osaka, Japan \\ Email: tanemura@derma.med.osaka-u.ac.jp
}

How to cite this paper: Tanemura, A., Takafuji, M., Hanaoka, Y., Kiyohara, E., Wataya-Kaneda, M. and Fujimoto, M. (2019) Successful Treatment of Intra-Arterial Peplomycin Infusion for Recurrent Oral Florid Papillomatosis. Journal of Cosmetics, Dermatological Sciences and Applications, 9, 223-227.

https://doi.org/10.4236/jcdsa.2019.93019

Received: June 25, 2019

Accepted: September 9, 2019

Published: September 12, 2019

Copyright $\odot 2019$ by author(s) and Scientific Research Publishing Inc. This work is licensed under the Creative Commons Attribution International License (CC BY 4.0).

http://creativecommons.org/licenses/by/4.0/ c) (i) Open Access

\begin{abstract}
A 56-year-old woman had noticed the erosion of oral mucosa and tongue 8 years ago. The mucosal lesions had been initially diagnosed as oral lichen planus and resistant to various treatments with prednisolone, etretinate and mizoribine and so on. One year ago, rapidly growing verrucous lesion occurred on her upper lip. Although we administered intralesional radiation therapy, the tumor recurred and new whitish lesions on the buccal mucosa and hard palate occurred 9 months after treatment. We confirmed an anatomical blood supply to the tumors by a fluorescent real-time imaging system and subsequently administered the intra-arterial infusion of peplomycin through retrograde catheters from bilateral superficial temporal arteries under the final diagnosis as oral florid papillomatosis (OFP). The tumors were dramatically shrunk and did not recur 16 months after treatment. OFP is known as clinically multiple whitish and verrucous lesions over the oral cavity and lip and a subtype of SCC with high differentiation. We suppose that an intra-arterial infusion therapy of peplomycin should be considered as the curative treatment for OFP.
\end{abstract}

\section{Keywords}

Oral Florid Papillomatosis, Selective Intra-Arterial Infusion, Peplomycin Sulfate

\section{Introduction}

Oral florid papillomatosis (OFP) is a clinicopathologic variant of a well-differentiated squamous cell carcinoma, verrucous carcinoma, and refers to a neoplastic lesion 
of the oral mucosa [1] [2]. The clinical course of OFP is commonly slow and localized without distant metastases, but relentless [2]. Although several treatment options are considered to manage OFP, most of them are often insufficient to cure. We present here an intractable case of OFP successfully treated with selective intra-arterial infusion of peplomycin.

\section{Case Presentation}

A 56-year-old woman noticed ulcerations on the buccal mucosa and tongue from around 2011. Although her tongue, upper and lower lip, and mucous membrane lesions were biopsied four times, no specific findings were found, so she was referred to our department in 2015. Upon an initial diagnosis as lichen planus, she had been administered with conservative oral drugs such as prednisolone, etretinate, mizoribine all of which were insufficient to cure. Even after we applied $60 \mathrm{~Gy} / 10 \mathrm{fr}$ of interstitial radiotherapy to the upper lip under the diagnosis as squamous cell carcinoma, the whitish plaque and concave expanded from the inside of the oral cavity to hard palate (Figure 1(a) and Figure 1(b)). Laboratory finding showed a slight increase in inflammatory response at CRP $0.33 \mathrm{mg} / \mathrm{dl}$ (normal; less than 0.2 ), or HbAlc $6.3 \%$ (normal ranged from 4.6 to 6.2), including no increase in tumor markers at SCC $1.7 \mathrm{ng} / \mathrm{ml}$ (normal; less than 2), and no other significant items were noted. No findings of lymph node metastasis or distant metastasis were observed upon contrast-enhanced CT. Histopathological findings on the upper lip at the time of recurrence revealed dense inflammatory cell infiltration in the upper dermis along with individual cell necrosis and mild cell atypia in the papillomatous elongated epidermis (Figure 1(c) and Figure 1(d)). The patient was finally diagnosed with oral florid
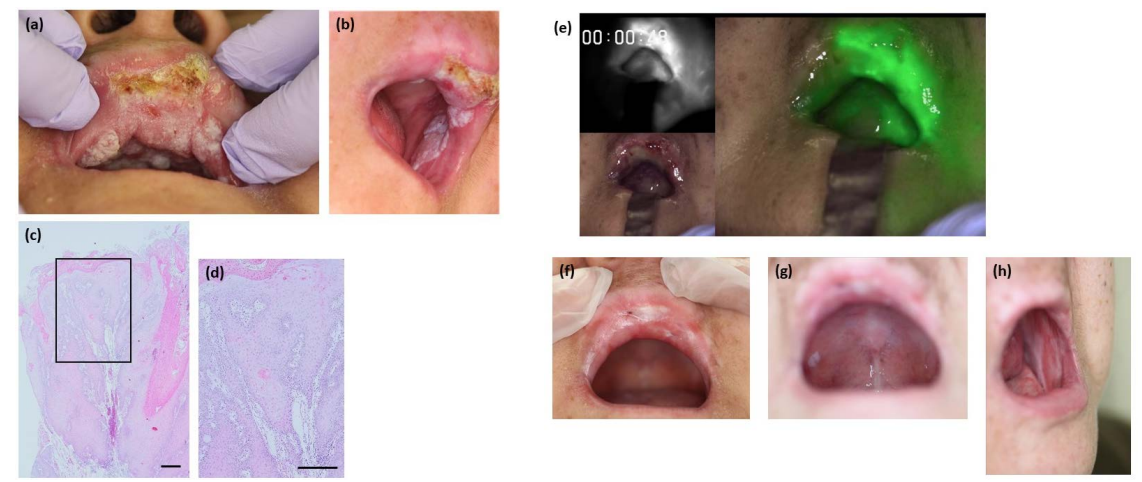

Figure 1. Clinical feature of multiple white-verrucous lesions overlying upper lid to hard palate (a) and left buccal mucosa (b); (c) Low power magnification view of $\mathrm{H}$ and $\mathrm{E}$ staining showed papillomatous hyperplasia of epidermis; (d) High power magnification view of the frame in (c) showed papillomatous highly differentiated squamous lesions proliferated into the dermis. The substantial cell atypia was mild. Each scale bar in (c) and (d) indicates $100 \mu \mathrm{m}$; (e) The live imaging system clearly visualized a blood supply from branches of the left external carotid artery. Three visions were simultaneously displayed and the upper left and right panel denote monochrome and green fluorescent view, respectively. Complete tumor remission on the upper lid (f); hard palate (g); and left buccal mucosa (h) was obtained 3 months after treatment. 
papillomatosis (OFP) based on the histopathological and clinical findings. We decided to apply a selective chemotherapy to cure multiple oral lesions all together. First, head angiography CT confirmed that the tumor was kept under control by the facial artery and mandibular artery which were branches of external carotid artery, thereafter, a catheter was retrogradely placed from the superficial temporal artery on both sides to the main carotid artery. A puncture was made from the superficial temporal artery, under general anesthesia, and once a catheter was placed in the external carotid artery, the catheter was fixed to the lateral temporal region at the position where the facial artery and the mandibular artery were imaged. Two weeks after placing the catheter, imaging with the near infrared light camera system was performed to confirm the imaging range of the catheter. Just 10 seconds after slowly injecting $1 \mathrm{ml}(5 \mathrm{mg})$ of indocyanine green fluorescent dye, it was confirmed by the live-captured camera that the region, which turned green on one side of the upper and lower lip buccal mucosa to the hard palate, covered the whole area of tumor lesions (Figure $1(\mathrm{e})$ ). Subsequently, peplomycin sulfate was administered daily on both sides, 5 $\mathrm{mg}$ each day for a total of 14 days $(10 \mathrm{mg} /$ day, for a total dose of $140 \mathrm{mg}$ ). Tumor necrosis began 4 days after initiation and all nodules in the lip and mouth disappeared 2 months after the completion of administration (Figures $1(f)-(h)$ ). No signs of recurrence and metastasis are observed 16 months after treatment.

\section{Discussion}

Oral florid papillomatosis (OFP) is a clinicopathologic variant of a well-differentiated squamous cell carcinoma, verrucous carcinoma, and refers to a neoplastic lesion of the oral mucosa [1] [2]. The clinical image indicates a characteristic cauliflower-like appearance, with a rough and white surface, and having a papillary shape. Smoking, poor oral hygiene, nonconforming dentures, and dental caries were considered as the cause of occurrence. In contrast, the evidence of HPV infection in OFP is still obscure [3]. There was no history of smoking and HPV staining was also negative in the present case. Because stomatitis occurred frequently after wearing dentures, a dental material and metal patch test was conducted and found positive for composite resin.

Although the first choice of treatment for OFP is surgical treatment, laser ablation treatment, cryosurgery treatment, treatment with retinoids, etc. are also considered [2]. Curative surgical treatment was extremely difficult in this case because the tumor was wholly located in the oral cavity from the lip and mouth where severe dysfunction after excision and complicated reconstruction were expected. While high dose radiation therapy presents another viable option, the risk of anaplastic transformation should be warranted after irradiation [4]. Since this case had already been treated with highly-concentrated intratumoral irradiation, we chose selective intra-arterial chemotherapy, which is a conservative treatment option. In the report of Fujimura et al., the intraoral injection of 20 to $110 \mathrm{mg}$ of peplomycin sulfate at a bolus dose of 5 to $10 \mathrm{mg} /$ day, for 24 squamous cell carcinomas of the lip, resulted in 3 cases of local recurrence and 3 cases of 
lymph node metastasis; however, it was reported that a complete response or partial response was maintained for 5 years in 19 cases [5]. Because peplomycin sulfate is a derivative of bleomycin, its antitumor effects are stronger than those of bleomycin and the blood concentration can be maintained for a long time, allowing the tissue concentration to be maintained high. Furthermore, the incidence of pulmonary fibrosis, which is mentioned as a serious side effect of bleomycin, is reported to be low in vivo [6]. Because the antitumor effect is known to be time-dependent, it is most effective to maintain the concentration above a certain level for a long time, with continuous administration most suitable as the administration method. It is acceptable that higher concentration of the drug can be obtained compared to intravenous injection therapy, as long as intra-arterial injection is administered via the external carotid artery [7].

Based on these findings, we conducted selective intra-arterial infusion therapy with peplomycin sulfate. Methods for intra-arterial injection of anticancer agents include 2 types; conventional method is to place a catheter in the external carotid artery and another is an intra-arterial injection from the femoral artery using the Seldinger method. While the Seldinger method can be used for reliable injection into the feeding artery of the tumor, the problem is that catheterization through the common carotid artery is required, potentially causing serious adverse events such as cerebrovascular disease and arterial rupture. Therefore, the conventional method was selected in this case, resulting in that insertion into a catheter was easy and continuous administration was possible.

\section{Conclusion}

It was possible to visualize arterially-controlled mucous membrane regions, as extremely clear and accurate real-time imaging. Furthermore, we were able to treat multiple squamous lesions, for which curative operation was not adequate, by intra-arterial infusion of peplomycin sulfate and archived almost no functional impairment after treatment. Referring to the present case, we emphasize that this selective chemotherapy could be a curable and preserved treatment option for OFP without surgical adaptation and that the real-time imaging system is practically useful to achieve an accurate delivery of peplomycin to the multiple OFP lesions.

\section{Author Contribution}

All authors participated in patient care, obtained patient history and workup, and wrote the manuscript.

\section{Conflicts of Interest}

The authors declare no conflicts of interest regarding the publication of this paper.

\section{References}

[1] Wechsler, H.L. and Fisher, E.R. (1962) Oral Florid Papillomatosis of the Oral Cavity 
and Larynx. Archives of Dermatology, 86, 480-492.

https://doi.org/10.1001/archderm.1962.01590100094019

[2] Schwartz, R.A. (1995) Verrucous Carcinoma of the Skin and Mucosa. Journal of the American Academy of Dermatology, 32, 1-21. https://doi.org/10.1016/0190-9622(95)90177-9

[3] Dias-Polak, D., Kra-Oz, Z., Szwarcwort-Cohen, M., Barzilai, A. and Bergman, R. (2019) A Case of Oral Florid Papillomatosis (Verrucous Carcinoma) with Lack of Evidence for Human Papillomavirus Involvement. The American Journal of Dermatopathology, 41, 617-619. https://doi.org/10.1097/00000372-900000000-98358

[4] Schwade, J.G., Wara, W.M., Dedo, H.H. and Phillips, T.L. (1976) Radiotherapy for Verrucous Carcinoma. Radiology, 120, 677-679. https://doi.org/10.1148/120.3.677

[5] Fujimura, T., Takahashi, K., Kambayashi, Y., Furudate, S., Hidaka, T., Kakizaki, A., Watabe, A., Haga, T., Hashimoto, A. and Aiba, S. (2017) Retrospective Investigation of Cutaneous Squamous Cell Carcinoma on the Lip Treated with Peplomycin Administered through a Superficial Temporal Artery. Anticancer Research, 37, 1885-1889. https://doi.org/10.21873/anticanres.11526

[6] Matsuda, A., Yoshioka, O., Takahashi, K., et al. (1978) Preclinical Studies on Bleomycin-PEP (NK-631) Bleomycin: Current Status and New Development. Academic Press Inc., Cambridge, MA, 311-331.

[7] Haga, T., Fujimura, T., Takeuchi, I., Deguchi, M. and Aiba, S. (2014) Successful Treatment of Two Cases of Squamous Cell Carcinoma on the Ear with Intra-Arterial Administration of Peplomycin through a Superficial Temporal Artery. Case Reports in Dermatology, 6, 207-212. https://doi.org/10.1159/000367804

\section{Abbreviation}

OFP: Oral Florid Papillomatosis 